\title{
Chronic Thromboembolic Pulmonary Hypertension Complicated by Giant Pulmonary Artery Aneurysm
}

\author{
Hiyo Obikane, ${ }^{1}$ MD, Mitsunobu Kaneko, ${ }^{2}$ MD, Masashi Kasao, ${ }^{2}$ MD, Hikaru Kumagai, ${ }^{1}$ MD, \\ Tomoyuki Tanino, ${ }^{1} \mathrm{MD}$ and Munehiro Yokoyama, ${ }^{1} \mathrm{MD}$
}

\begin{abstract}
Summary
A pulmonary artery (PA) aneurysm is an extremely rare condition that can be idiopathic or secondary. Only a few reports on giant PA aneurysms associated with chronic thromboembolic pulmonary hypertension (CTEPH) are available in the literature. Here, we present a case of CTEPH associated with a secondary giant PA aneurysm detected by autopsy. A 68-year-old woman was diagnosed with pulmonary hypertension (PH) and a PA aneurysm with a diameter of $7.5 \mathrm{~cm} 7$ years before admission. CTEPH was suspected as the cause of PH; however, she refused to undergo surgical treatment. Although her condition improved temporarily with pulmonary vasodilators, she had recurrence of heart failure and died because of the deterioration of her general condition. An autopsy revealed a giant PA aneurysm without medial degeneration, suggesting a secondary PA aneurysm associated with $\mathrm{PH}$. Histological findings indicated multiple organized thrombi with recanalization in the PA bilaterally, and CTEPH was diagnosed as the cause of PH. Although rare, when a PA aneurysm is detected, it is important to consider that CTEPH might be associated with a giant PA aneurysm. A better understanding of this condition is necessary to improve the therapeutic strategy.
\end{abstract}

(Int Heart J 2021; 62: 938-943)

Key words: Right-sided heart failure, Cardiac hypertrophy, Vasodilators, Autopsy

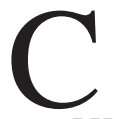

hronic thromboembolic pulmonary hypertension (CTEPH) is one of the major causes of pulmonary hypertension $(\mathrm{PH})$ and right-sided heart failure. $\mathrm{PH}$ is known to cause secondary pulmonary artery (PA) aneurysms; however, there are very few reports in the literature regarding the association of CTEPH with giant PA aneurysms. ${ }^{1,2)}$ PA aneurysm is an extremely rare pathological condition. Although surgical indications have been discussed in the literature, to date, a clear consensus regarding treatment has not yet been established., ${ }^{3,4)}$ Therefore, it is necessary to consider the diagnosis and treatment strategy for CTEPH complicated by a PA aneurysm. Here, we report on a rare case of CTEPH associated with a giant PA aneurysm detected by autopsy and present a review of the literature.

\section{Case Report}

The patient, a 68-year-old woman, was diagnosed with hypertension and cardiac hypertrophy 11 years prior to her admission. Seven years before admission, she was referred to our hospital with a complaint of dyspnea on exertion. An examination revealed the presence of PH (PA pressure, $86 / 32$ (53) $\mathrm{mmHg}$; total pulmonary vascular resistance, 613.3 dyne $*$ seconds $* \mathrm{~cm}^{-5}$ ). Her mean PA wedge pressure was elevated to $20 \mathrm{mmHg}$, which subsequently normalized to $12 \mathrm{mmHg}$ after treatment for left heart failure. However, her PH persisted, and she was diagnosed with pulmonary arterial hypertension. Computed tomography $(\mathrm{CT})$ revealed a PA aneurysm with a diameter of $7.5 \mathrm{~cm}$ and thromboembolism in both main trunks of the PA (Figures 1A, 2A). Lung perfusion scintigraphy showed wedge-shaped defects on both lungs (Figure 2B). Laboratory data revealed an increased D-dimer level (1.5 $\mu \mathrm{g} / \mathrm{mL}$ ) but a normal C-reactive protein (CRP) level $(0.1$ $\mathrm{mg} / \mathrm{dL}$ ). Therefore, CTEPH was suspected as the cause of $\mathrm{PH}$, and anticoagulant therapy and treatment for heart failure were initiated. Although surgical treatment was recommended, the patient refused to undergo surgery. Unfortunately, balloon pulmonary angioplasty (BPA) could not be performed because of patient refusal and the risk of rupture of the PA aneurysm due to catheter treatment. Hence, treatment with vasodilators (prostacyclin analogs, endothelin receptor antagonists, and phosphodiesterase [PDE5] inhibitors) and home oxygen therapy were initiated. The treatment led to temporarily improved symptoms; however, exacerbation and remission of heart failure were repeatedly observed. Figure 3 illustrates the course of the right ventricular systolic pressure (RVSP), which represents the estimated PA systolic pressure that was meas-

From the ${ }^{1}$ Department of Diagnostic Pathology, Tokyo Metropolitan Police Hospital, Tokyo, Japan and ${ }^{2}$ Department of Cardiology, Tokyo Metropolitan Police Hospital, Tokyo, Japan.

Address for correspondence: Hiyo Obikane, MD, Department of Diagnostic Pathology, Tokyo Metropolitan Police Hospital, 4-22-1 Nakano, Nakano-ku, Tokyo, 164-8541, Japan. E-mail: hdoi03@ybb.ne.jp

Received for publication August 13, 2020. Revised and accepted January 6, 2021.

Released in advance online on J-STAGE July 6, 2021.

doi: 10.1536/ihj.20-572

All rights reserved by the International Heart Journal Association. 
A

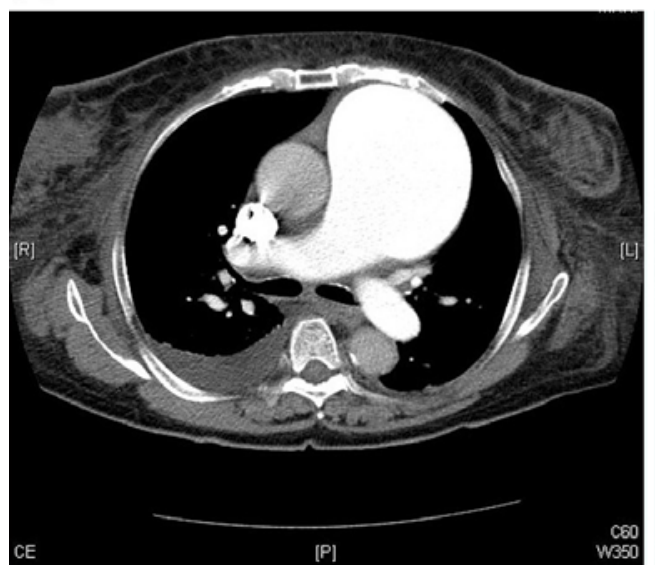

B

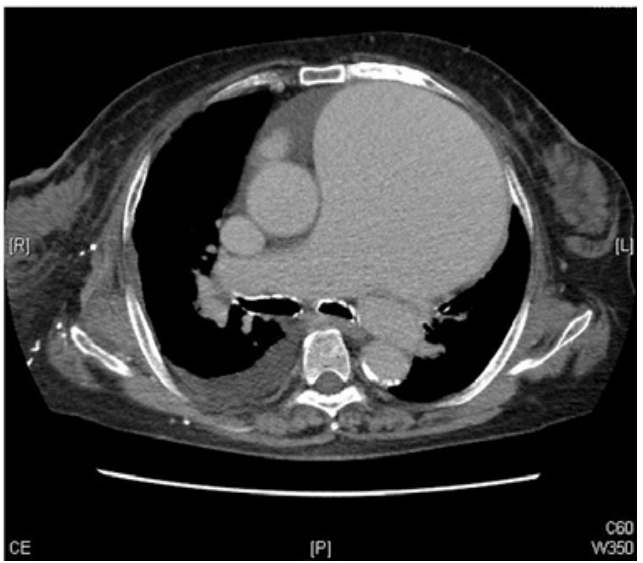

Figure 1. Computed tomography (CT) 7 years before admission (A) and on admission (B). A: Contrast-enhanced CT shows a dilated pulmonary artery (PA) with a diameter of approximately $7.5 \mathrm{~cm}$. B: On admission, the PA was dilated to a diameter of approximately $12 \mathrm{~cm}$.

A

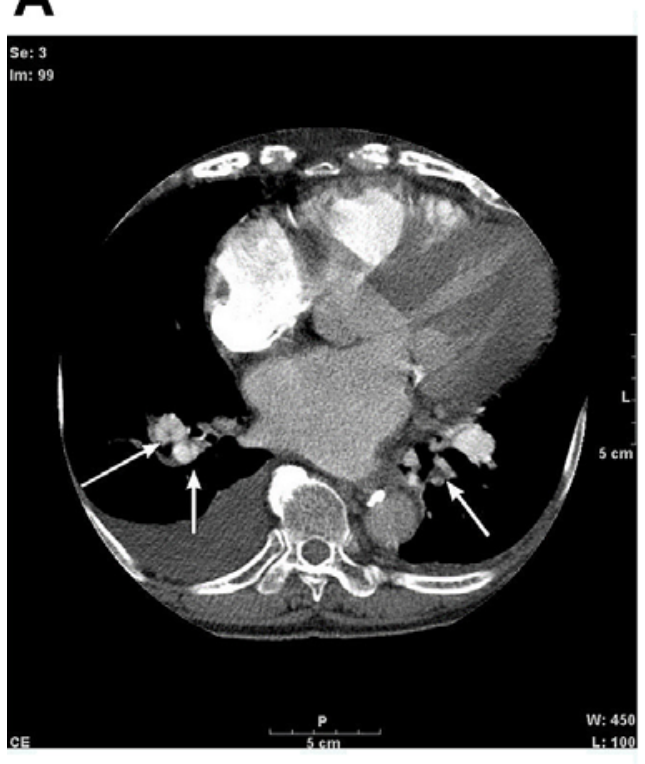

B

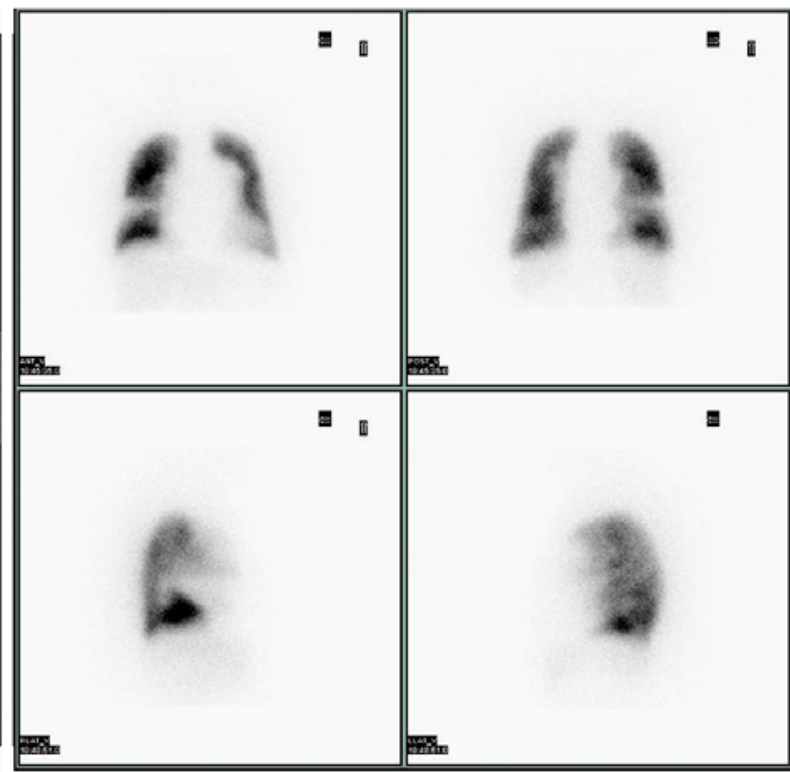

Figure 2. Computed tomography (CT) (A) and lung perfusion scintigraphy (B) 7 years before admission. A: Multiple thrombi on both sides of the central pulmonary artery (PA) are detected (arrow). B: A wedge-shaped blood flow defect is observed at the thrombus site (bilateral lower lungs).

ured by echocardiography and the diameter of the PA aneurysm after the first admission. Ninety-four days before her death, she was urgently admitted to our hospital because of progression of right-sided heart failure due to PH. On admission, laboratory data indicated an elevated brain natriuretic peptide level $(648.1 \mathrm{pg} / \mathrm{mL})$, normal CRP level $(0.1 \mathrm{mg} / \mathrm{dL})$, and normal D-dimer level $(0.20 \mu \mathrm{g} /$ $\mathrm{mL})$. Chest CT indicated an increase in the diameter of the PA aneurysm from 7.5 to $12 \mathrm{~cm}$ (Figure 1B). The estimated PA systolic pressure was $68.7 \mathrm{mmHg}$, according to echocardiography.

Although the administration of human atrial natriuretic peptide and diuretics was initiated, there was no im- provement in the heart failure. Soluble guanylyl cyclase (sGC) was introduced, instead of the PDE-5 inhibitor, on day 78; however, the patient's general condition did not improve, and renal dysfunction was observed. Therefore, sGC was discontinued on day 93. The patient died the next day.

An autopsy was performed 25 hours after the patient's death after obtaining informed consent from her family. Upon opening of the chest, a saccular dilated PA was confirmed (Figure 4A). The heart weighed $790 \mathrm{~g}$, and right ventricular dilation and bilateral hypertrophy were observed (Figure 4B and C). The PA aneurysm circumference was $15 \mathrm{~cm}$, and the pulmonary valve circumference 


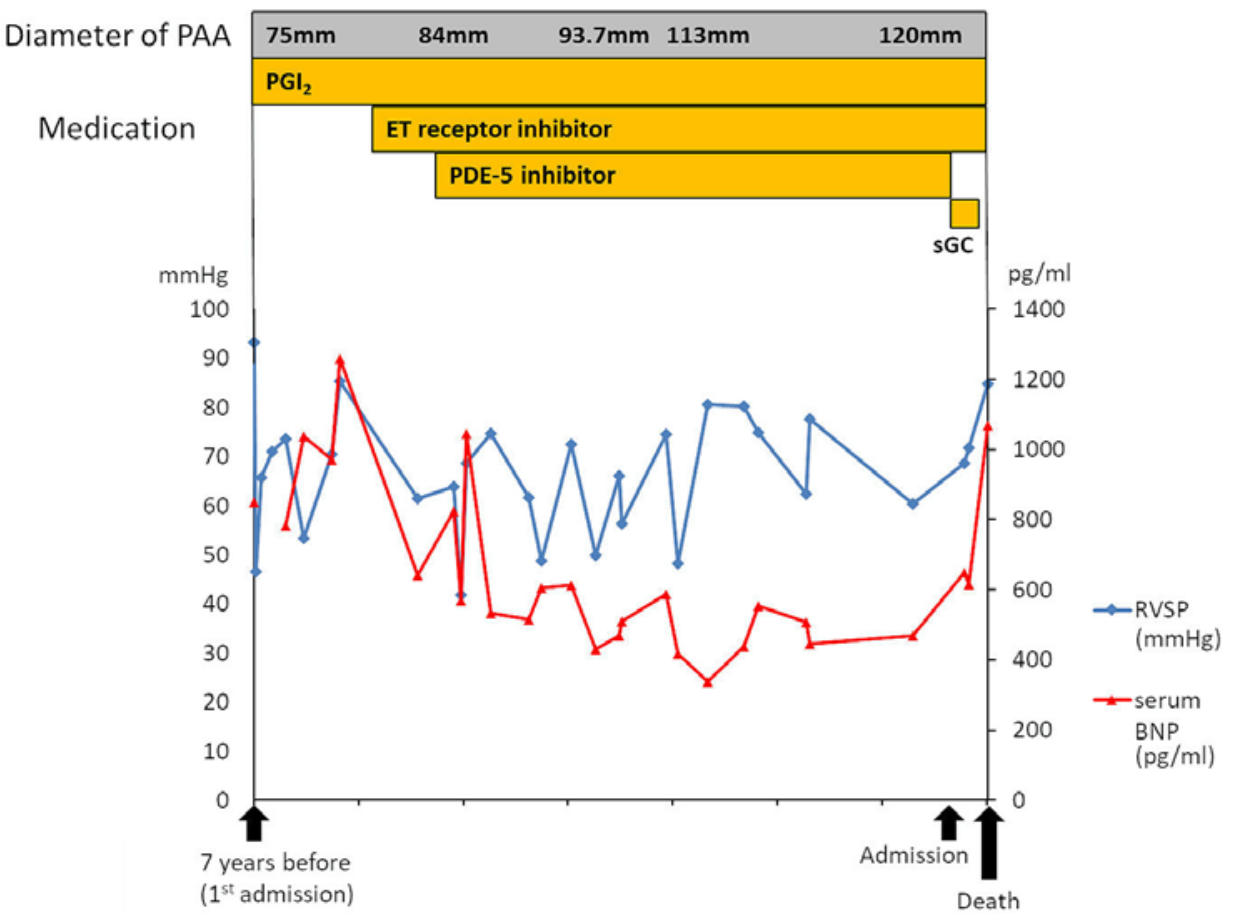

Figure 3. The patient's clinical course in response to the administered medications was monitored using measurements of right ventricular systolic pressure (RVSP), serum brain natriuretic peptide (BNP) concentration, and diameter of the pulmonary artery aneurysm (PAA). The RVSP represents the estimated pulmonary artery's systolic pressure and was measured using echocardiography. Despite long-term use of various pulmonary vasodilators, the RVSP remained generally high, and the diameter of the PAA gradually increased over 7 years. The administered medications included prostacyclin analogs, endothelin receptor antagonists, phosphodiesterase inhibitors, and soluble guanylate cyclase.
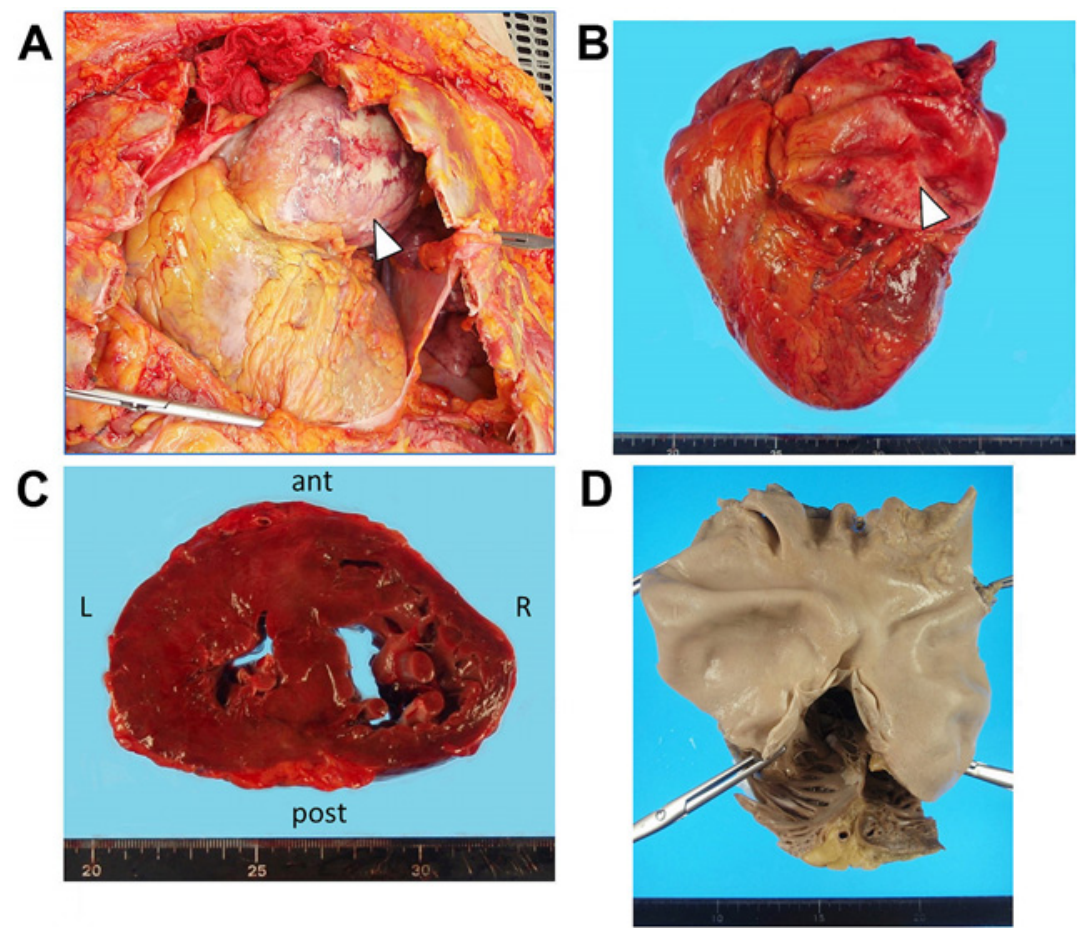

Figure 4. Gross findings of the autopsy examination. A: A pulmonary artery (PA) aneurysm was confirmed when the chest was opened (arrowhead). B: The excised heart with the PA aneurysm (arrowhead). C: Cross-section of the heart showing hypertrophy and dilation of both ventricles. R indicates right; L, left; ant, anterior; and post, posterior. D: Lumen of the PA. 

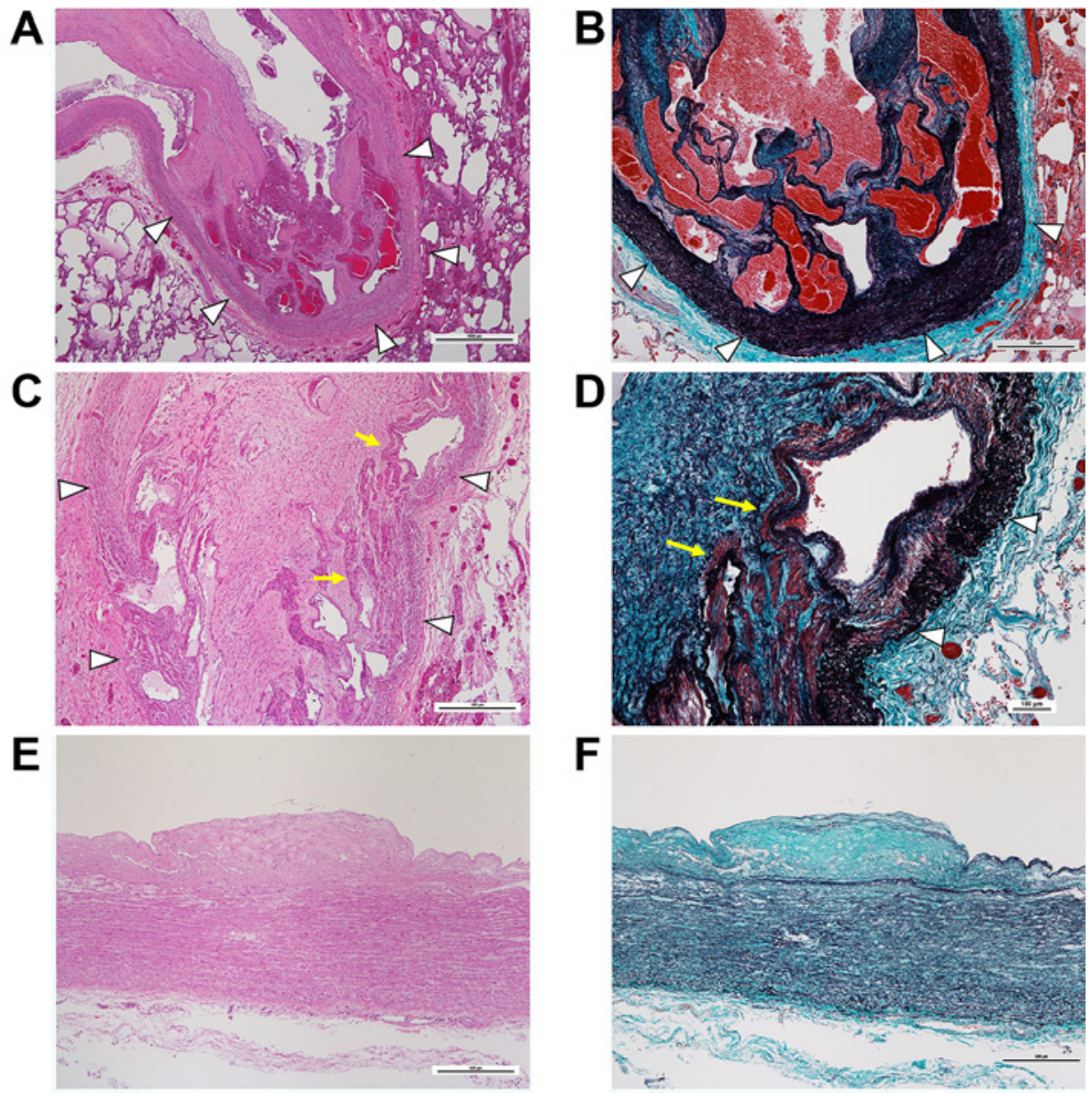

Figure 5. Histopathological findings of the pulmonary artery (PA) in hematoxylin-eosin staining (A, C, E) and Elastica Masson Goldner (EMG) staining (B, D, F). A-D: An organized thrombus with recanalization is found in the lumen of the PA. The arrowhead indicates the media of the PA. The recanalized vessels are surrounded by smooth muscle cells (yellow arrow). The PA in the left hilar region (diameter: $3,000 \mu \mathrm{m})(\mathbf{A}, \mathbf{B})$. The first branch of the PA in the right hilar region (diameter: 2,300 $\mu \mathrm{m})(\mathbf{C}, \mathbf{D})$. E: Atherosclerotic change was recognized in the intima of the PA aneurysmal wall. F: EMG staining of the PA aneurysmal wall without cystic medial degeneration.

was $9 \mathrm{~cm}$ (Figure 4D). No evidence of PA stenosis was found. A histopathological examination revealed multiple organized thrombi with recanalization vessels (so-called 'colander-like' lesions) in both branches of the PA (Figure 5A-D). Focal atherosclerotic plaque was observed in the aneurysm, but no cystic medial degeneration was found (Figure 5E and F). Because there was no apparent complication of medial degeneration, the PA aneurysm was diagnosed as secondary to PH. The heart had moderate hypertrophy of myocytes and mild fibrosis, but no disarray was found. Therefore, it was assumed that the patient had hypertensive heart disease rather than hypertrophic cardiomyopathy (HCM). Additionally, multi-organ congestion, pleural effusion, and ascites associated with heart failure were observed. Autopsy findings indicated that the direct cause of death was CTEPH and right-sided and left-sided heart failure.

\section{Discussion}

We reported the case of a 68-year-old woman who died after exacerbation of heart failure due to CTEPH with a giant PA aneurysm. CTEPH is a pathological condition with a poor prognosis that causes chronic PA occlusion or stenosis due to an organized thrombus in the PA. Conservative and surgical treatments are currently available for CTEPH. Pulmonary thromboendarterectomy (PTE) and BPA are known as effective surgical treatments for CTEPH that can improve the prognosis. ${ }^{5,6}$

In this case, a large PA aneurysm was identified at the time when $\mathrm{PH}$ was diagnosed; however, the clinical course was not clear, and it was difficult to determine whether the patient's PH and PA aneurysm were caused by CTEPH alone. The differential diagnoses included CTEPH with idiopathic PA aneurysms and CTEPH with HCM.

Histopathologically, many colander-like lesions were found in the PA; these findings were consistent with 
Table. Classification of Pulmonary Artery Aneurysms and Pathological Findings of Idiopathic Pulmonary Artery Aneurysms

\begin{tabular}{ll}
\hline \multicolumn{2}{l}{ Causes of pulmonary artery aneurysms } \\
\hline $\begin{array}{l}\text { Idiopathic } \\
\text { Congenital }\end{array}$ & Absence of cardiac or pulmonary disease (Pathological findings) Simple dilatation or medial degeneration \\
Heart defect & Persistent ductus arteriosus, Ventricular septal defects, Atrial septal defects, Hypoplastic aortic valve, \\
& Bicuspid aortic valve, Pulmonary valve stenosis, Pulmonary regurgitation, Absent pulmonary valve \\
Connective tissue abnormalities & Ehlers-Danlos syndrome, Marfan syndrome, Cystic medial necrosis \\
Acquired & \\
Infectious & Syphilis, Tuberculosis, Pyogenic bacteria, Septic embolisms, Bacterial and fungal pneumonia \\
Vasculitis & Behcet syndrome, Hughes-Stovin syndrome \\
Pulmonary arterial hypertension & \\
$\begin{array}{l}\text { Chronic pulmonary embolism } \\
\text { Neoplasm }\end{array}$ & Primary lung cancer, Pulmonary metastasis \\
Iatrogenic & Cardiac surgery, Catheters, Chest tubes, Angiography, Surgical resection, Biopsy \\
\hline
\end{tabular}

This table has been adapted from Kreibich, et al. ${ }^{8)}$

CTEPH. A previous report mentioned that intimal thickening, similar to that observed with idiopathic PA hypertension (IPAH), can be noted in the peripheral blood vessels of CTEPH cases $^{7}$; however, no such findings were observed in this case.

The Table presents the classification of PA aneurysms and pathological findings of idiopathic PA aneurysms. PA aneurysms are classified as idiopathic or secondary. ${ }^{8)}$ Idiopathic PA aneurysms have no underlying disease and may be accompanied by medial degeneration of the PA. ${ }^{9}$ Our patient had PH in the background of a PA aneurysm, but the clinical course was unknown, and it could not be ruled out that an idiopathic PA aneurysm may have preceded it. However, histological findings suggestive of medial degeneration were not found with the PA aneurysm; hence, an idiopathic PA aneurysm was not considered. ${ }^{9)}$

Although biventricular hypertrophy was observed, disarray of the myocardium could not be confirmed. Therefore, in this case, we determined that cardiac hypertrophy was associated with $\mathrm{PH}$ and hypertension and that it was not a complication of HCM.

$\mathrm{PH}$ is an important cause of secondary PA aneurysms. PA aneurysms caused by $\mathrm{PH}$ have been reported to progress and expand over time, regardless of the improvement in PA pressure. ${ }^{10)}$ The patient in this case presented with a giant PA aneurysm when PH was diagnosed; however, the prior clinical course could not be confirmed. Therefore, it was particularly difficult to establish a PA aneurysm as the clinical diagnosis.

For PA aneurysms, the indication for surgery is determined by the size of the aneurysm and the presence or absence of $\mathrm{PH}$ or congenital heart disease. ${ }^{3)}$ The patient in this case was a candidate for surgery but refused to undergo surgery. When a PA aneurysm is complicated by IPAH, PA replacement should be considered to prevent the rupture of the aneurysm; surgical treatment, including heart and lung transplantation, may also be required. ${ }^{11)}$ There have been only a few reports on the association between CTEPH and giant PA aneurysms. ${ }^{1,2)}$ However, because the prognosis of CTEPH has improved because of PTE and BPA in recent years, these treatments are likely to improve $\mathrm{PH}$ and the resulting right-sided heart failure.

In conclusion, the treatment strategy for PA aneurysms varies depending on the cause of $\mathrm{PH}$. Therefore, when a giant PA aneurysm is confirmed, the cause should be carefully considered. Although rare, when a PA aneurysm is observed, it is important to consider that CTEPH might be associated with a giant PA aneurysm.

\section{Disclosure}

Conflicts of interest: The authors declare that there is no conflict of interest.

\section{References}

1. Heikkinen J, Milger K, Alejandre-Lafont E, et al. Cardiovocal syndrome (Ortner's syndrome) associated with chronic thromboembolic pulmonary hypertension and giant pulmonary artery aneurysm: Case report and review of the literature. Case Rep Med 2012; 2012: 230736.

2. Nesta M, Cammertoni F, Mangini S, Colizzi C, Bruno P, Massetti M. Angina in left main coronary artery occlusion by pulmonary artery aneurysm. Asian Cardiovasc Thorac Ann 2017; 25: 216-8.

3. Duijnhouwer AL, Navarese EP, Van Dijk AP, Loeys B, RoosHesselink JW, De Boer MJ. Aneurysm of the pulmonary artery, a systematic review and critical analysis of current literature. Congenit Heart Dis 2016; 11: 102-9.

4. Hou R, Ma GT, Liu XR, et al. Surgical treatment of pulmonary artery aneurysm: an institutional experience and literature review. Interact Cardiovasc Thorac Surg 2016; 23: 438-42.

5. Moser KM, Daily PO, Peterson K, et al. Thromboendarterectomy for chronic, major-vessel thromboembolic pulmonary hypertension. Immediate and long-term results in 42 patients. Ann Intern Med 1987; 107: 560-5.

6. Tanabe N, Kawakami T, Satoh T, et al. Balloon pulmonary angioplasty for chronic thromboembolic pulmonary hypertension: a systematic review. Respir Investig 2018; 56: 332-41.

7. Dorfmüller P, Günther S, Ghigna MR, et al. Microvascular disease in chronic thromboembolic pulmonary hypertension: a role for pulmonary veins and systemic vasculature. Eur Respir J 2014; 44: 1275-88.

8. Kreibich M, Siepe M, Kroll J, Höhn R, Grohmann J, Beyersdorf F. Aneurysms of the pulmonary artery. Circulation 2015; 131: 310-6.

9. Deb SJ, Zehr KJ, Shields RC. Idiopathic pulmonary artery aneurysm. Ann Thorac Surg 2005; 80: 1500-2.

10. Akagi S, Nakamura K, Sarashina T, Ejiri K, Kasahara S, Ito H. Progression of pulmonary artery dilatation in patients with pulmonary hypertension coexisting with a pulmonary artery aneu- 
rysm. J Cardiol 2018; 71: 517-22.

11. Shayan H, Sareyyupoglu B, Shigemura N, Thacker J, Bermudez

C, Toyoda Y. Lung transplant, double valve repair, and pulmo- nary artery aneurysm resection. Ann Thorac Surg 2012; 93: e35 . 